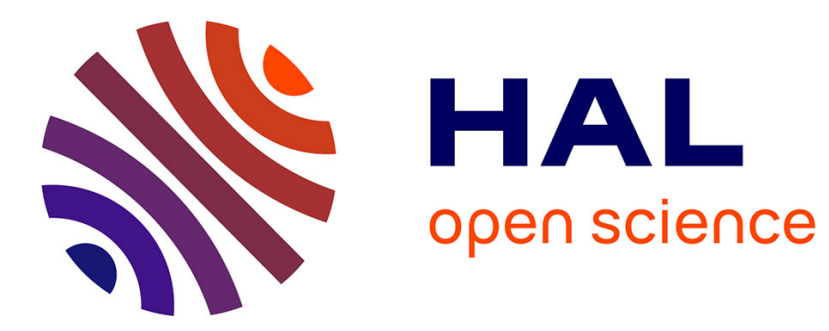

\title{
Réalisation d'un densitomètre différentiel
}

J.C. Merle, C. Wecker

\section{To cite this version:}

J.C. Merle, C. Wecker. Réalisation d'un densitomètre différentiel. Revue de Physique Appliquée, 1975, 10 (4), pp.221-225. 10.1051/rphysap:01975001004022100 . jpa-00243907

\section{HAL Id: jpa-00243907 https://hal.science/jpa-00243907}

Submitted on 1 Jan 1975

HAL is a multi-disciplinary open access archive for the deposit and dissemination of scientific research documents, whether they are published or not. The documents may come from teaching and research institutions in France or abroad, or from public or private research centers.
L'archive ouverte pluridisciplinaire HAL, est destinée au dépôt et à la diffusion de documents scientifiques de niveau recherche, publiés ou non, émanant des établissements d'enseignement et de recherche français ou étrangers, des laboratoires publics ou privés. 


\author{
Classification \\ Physics Abstracts \\ 0.640
}

\title{
RÉALISATION D'UN DENSITOMĖTRE DIFFÉRENTIEL
}

\author{
J. C. MERLE et C. WECKER \\ Laboratoire de Spectroscopie et d'Optique du Corps Solide $\left(^{*}\right)$ \\ Université Louis-Pasteur, 5, rue de l'Université, 67000 Strasbourg, France
}

(Reçu le 3 décembre 1974, accepté le 19 mars 1975)

\begin{abstract}
Résumé. - Nous décrivons un densitomètre différentiel, réalisé avec des moyens courants de laboratoire. Il permet d'obtenir simultanément la courbe densitométrique d'un spectre et sa dérivée par rapport à la longueur d'onde. Les avantages et les performances de cet appareil sont discutés. A titre d'exemple, nous présentons quelques résultats expérimentaux obtenus sur le spectre d'absorption de $\mathrm{Cu}_{2} \mathrm{O}$.
\end{abstract}

Abstract. - We describe a differential densitometer, which can be easily constructed with the usual resources of a laboratory. It enables to obtain both the densitometric curve of a spectrum and its derivative with respect to the wavelength. The advantages and performances of this apparatus are discussed. As an example we give some experimental results obtained for the absorption spectrum of $\mathrm{Cu}_{2} \mathrm{O}$.

Introduction. - Nous avons été amenés à réaliser un densitomètre différentiel pour améliorer l'analyse des photographies de spectres magnéto-optiques. Cet appareil permet d'obtenir simultanément des représentations du spectre et de sa dérivée par rapport à la longueur d'onde. Il apporte des renseignements plus complets qu'un densitomètre normal, mais nécessite des plaques à grains fins.

Nous discuterons certains avantages des mesures en modulation de longueur d'onde, puis nous décrirons le principe, la réalisation et les performances de notre appareil. Son utilisation pour d'autres mesures, en particulier la chromatographie, sera évoquée.

1. Modulation de la longueur d'onde. - L'étude spectrographique en modulation de longueur d'onde présente une sensibilité incomparable pour la détection de structures peu prononcées [1] (raies et bords d'absorption peu intenses par exemple). Dans de nombreux montages spectrophotométriques, la modulation est obtenue, soit en faisant vibrer l'une des fentes d'un monochromateur, soit en déviant légèrement le faisceau, avant la fente de sortie, à l'aide d'un miroir ou d'une lame vibrante [2]. La réalisation de ces montages est particulièrement délicate lorsqu'on désire conserver toute la résolution de l'appareil dispersif. D'autre part,

(*) Laboratoire associé au C. N. R. S. n 232. lors des mesures d'absorption ou de réflexion, des sources lumineuses très brillantes sont nécessaires. Mais ces dernières fournissent généralement des spectres d'émission très structurés et imposent l'utilisation d'un montage à deux voies, onéreux et complexe [3].

Des résultats comparables à ceux d'une étude spectrographique en modulation de la longueur d'onde, peuvent être obtenus de façon simple à partir d'un densitométrage différentiel (pseudo-modulation de la longueur d'onde).

La non-linéarité des plaques photographiques empêche toute mesure quantitative précise; ce fait est sans importance dans la mesure où l'on s'intéresse à la position des structures et non à leur intensité. Par contre, on bénéficie des avantages inhérents à la détection photographique : rendement quantique élevé, choix du temps d'exposition, informations simultanées sur la totalité d'un spectre. Comparée à une détection utilisant un photodétecteur unique, la détection photographique est donc avantageuse pour l'étude de spectres compliqués, contenant un nombre important d'informations.

L'utilisation d'un densitomètre s'impose également dans d'autres domaines que la spectroscopie. C'est le cas, par exemple, de la chromatographie (chromatographie sur plaques, électrophorèse, etc.). Les échantillons que l'on analyse présentent alors des raies 
(ou bandes) parallèles et diversement colorées apparaissant sur un fond transparent (gel par exemple) ou absorbant (silice en poudre par exemple). Le densitométrage permet d'obtenir l'absorption $K$ en fonction de la distance $x$ à une ligne de référence. La connaissance de la dérivée $\frac{\mathrm{d}}{\mathrm{d} x} K(x)$ est utile dans tous les cas où $K(x)$ présente des fonds continus que l'on cherche à éliminer et dans les cas où l'on veut résoudre des raies peu intenses et mal séparées de raies (ou bandes) plus larges et plus intenses.

2. Principe de l'appareil. - Rappelons le principe d'un densitomètre normal : l'image d'une fente est focalisée sur la plaque photographique à analyser et la lumière transmise est recueillie sur un photodétecteur. Un système mécanique translate la plaque à vitesse constante, perpendiculairement à l'image de la fente. On enregistre l'information délivrée par le photodétecteur en fonction du temps; on obtient ainsi une représentation $I(\lambda)$ du spectre étudié (intensité lumineuse $I$ transmise par la plaque en fonction de la longueur d'onde $\lambda$ ). On détermine généralement les longueurs d'onde en superposant le spectre à étudier et un spectre d'émission de raies de référence.

Projetons sur le film deux images de fentes identiques, parallèles et également éclairées $F_{1}$ et $F_{2}$, et recueillons séparément les flux transmis par deux photodétecteurs $\mathrm{D}_{1}$ et $\mathrm{D}_{2}$. On enregistrera alors les intensités transmises $I_{1}=I\left(\lambda_{1}\right)$ et $I_{2}=I\left(\lambda_{2}\right)$. Si la distance entre les images $F_{1}$ et $F_{2}$ tend vers zéro, la quantité $I_{1}-I_{2}$ est proportionnelle à la dérivée du spectre par rapport à la longueur d'onde. En pratique, on utilisera des images $F_{1}$ et $F_{2}$ jointives et on pourra mesurer simultanément le spectre $\left(I_{1}\right.$ ou $\left.I_{2}\right)$ et sa dérivée. Un tel système offre les avantages d'une mesure différentielle : détermination précise de faibles quantités avec un ensemble de mesures de stabilité moyenne. En conséquence, il permet d'obtenir de meilleures performances qu'une dérivation numérique, effectuée après une mesure classique de $I(\lambda)$. Par ailleurs, l'utilisation de deux fentes jointives de largeur $a$ est plus avantageuse que l'utilisation d'une fente de largeur $a$ vibrant avec l'amplitude totale $a$. En effet, on obtient, dans les deux cas, des spectres dérivés de qualité comparable, mais le spectre normal est analysé avec une meilleure résolution avec un système à fentes fixes.

3. Réalisation. - Le schéma de notre densitomètre différentiel est donné par la figure 1. Une fente $F$ d'ouverture et d'inclinaison réglables est éclairée au moyen de la source $\mathrm{S}$ (lampe à ruban de tungstène). Deux images de cette fente, $F_{1}$ et $F_{2}$, sont focalisées dans le plan du film à analyser. A cet effet, on utilise un prisme de Wollaston $\mathrm{W}$, qui divise un faisceau de lumière naturelle et parallèle en deux faisceaux légèrement divergents et totalement polarisés dans des directions complémentaires. Les images $F_{1}$ et $F_{2}$ sont donc polarisées. Leur écart peut être réglé en choisis-

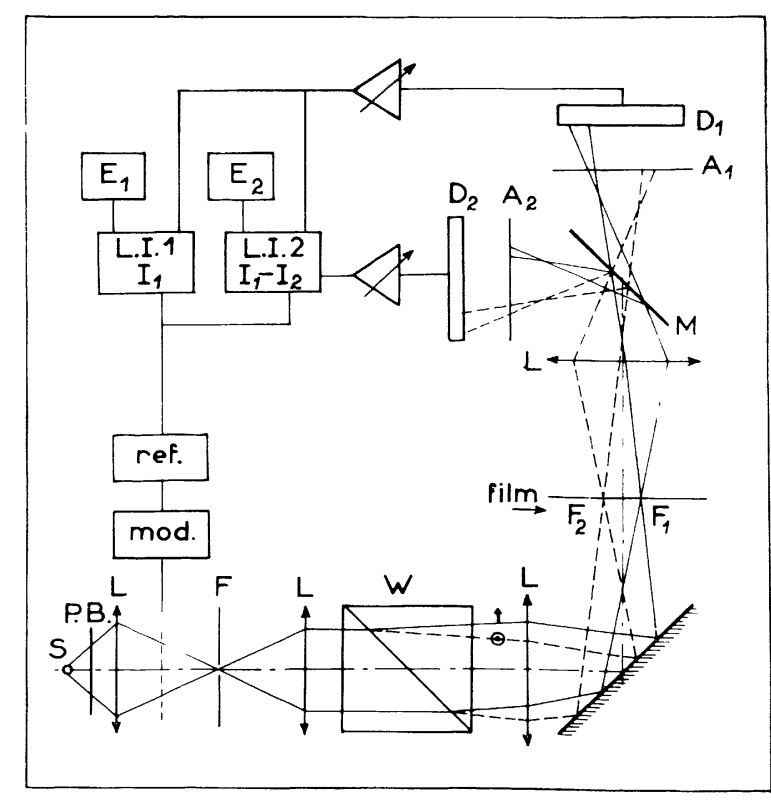

FIG. 1. - Schéma du densitomètre différentiel.

sant les focales des lentilles et en jouant sur l'orientation du prisme W.

Les faisceaux lumineux transmis par la plaque photographique sont ensuite séparés par le dispositif $M$; sur chacun des faisceaux, les analyseurs $A_{1}$ et $A_{2}$ permettent de sélectionner les flux, selon leur polarisation, pour les transmettre aux photodétecteurs $\mathrm{D}_{1}$ et $\mathrm{D}_{2}$.

L'essentiel du montage a été réalisé à partir d'un microscope Zeiss binoculaire à objectif unique. Sa platine sert de porte-plaque et son optique permet de séparer les faisceaux. Chacun des oculaires a été muni d'un analyseur et d'un photodétecteur. La platine d'origine étant munie d'une vis micrométrique, nous y avons adapté un châssis servant de porte-plaque. Son mouvement uniforme est assuré par un moteur synchrone accouplé à la vis micrométrique.

Le montage optique comprend, en outre, un filtre passe bande (P. B.) sélectionnant la partie rouge du spectre de la source $\mathrm{S}$ : dans cette région, les polariseurs et analyseurs utilisés sont efficaces et le rendement des détecteurs est important. Un disque tournant (mod.) permet une modulation totale de l'intensité lumineuse à la fréquence de $800 \mathrm{~Hz}$. La partie détection comprend des photodiodes au silicium S. G. D. 100 de $E G \&$ \&, dont la réponse est parfaitement linéaire pour sept décades de puissance lumineuse. Elles sont suivies de deux préamplificateurs à gain variable, qui permettent d'équilibrer les signaux issus de $D_{1}$ et $D_{2}$ en l'absence de film. Deux amplificateurs synchrones suivis de deux enregistreurs fournissent simultanément les quantités $I_{1}$ et $I_{1}-I_{2}$.

Les photodétecteurs étant amovibles, le microscope peut être rendu à son usage normal en quelques secondes. D'autre part, les réglages préliminaires à tout 
densitométrage : mise au point sur le film, largeur de fente, position respective des images des fentes et parallélisme avec les raies de référence, se font par observation à travers les oculaires.

Ces réglages sont aisés et plus précis que sur la plupart des densitomètres commerciaux.

La majorité des éléments que nous utilisons était en notre possession et sert à d'autres fins. En particulier, les amplificateurs synchrones et les enregistreurs appartiennent à une autre chaîne de mesures. On pourrait se contenter d'un simple amplificateur différentiel et d'un seul enregistreur pour détecter successivement $I_{1}$ puis $I_{1}-I_{2}$.

4. Performances. - Dans les mesures habituelles de spectrophotométrie, la sensibilité d'un montage est limitée par les bruits prenant naissance au niveau du photodétecteur. La précision des mesures est directement liée au temps de mesure (intégration temporelle) et à la résolution qui détermine le flux reçu par le photodétecteur.

Un densitomètre utilise généralement des flux lumineux assez élevés et le bruit au niveau du photodétecteur est négligeable. La qualité d'un densitométrage est essentiellement limitée par les bruits spatiaux, introduits par les irrégularités des plaques photographiques, c'est-à-dire leur grain. Il faut donc utiliser des plaques grain fin et des fentes de grande surface réalisant une intégration sur un nombre important de grains. Ces conditions impliquent, lors de la photographie des spectres, des temps d'exposition élevés (plaque à grain fin $=$ plaque lente) et une résolution limitée (largeur de la fente du spectrographe); elles devront être impérativement respectées pour les densitométrages différentiels où l'on cherche à détecter des structures peu prononcées.

Dans nos conditions habituelles de travail, les spectres sont obtenus à partir d'un spectrographe Bausch \& Lomb de distance focale $2 \mathrm{~m}$, muni d'un réseau de $2400 \mathrm{tr} / \mathrm{mm}$, et ayant une dispersion linéaire de $2 \AA / \mathrm{mm}$. Sa résolution optimale est atteinte avec des fentes de 10 à $20 \mu \mathrm{m}$.

Nous l'utilisons généralement avec des fentes de $50 \mu \mathrm{m}$ et nous effectuons les densitométrages avec des fentes jointives de même largeur. Le spectre densitométré est ainsi obtenu avec une résolution de $0,1 \AA$ et le spectre dérivé avec une pseudo-modulation de $0,1 \AA$ d'amplitude.

Le microscope constituant le densitomètre est utilisé avec un objectif $\times 2,5$ et deux oculaires $\times 10$ : son champ limite la hauteur utile des fentes à $5 \mathrm{~mm}$. Sa résolution est de $5 \mu \mathrm{m}$.

5. Résultats expérimentaux. - Nous avons utilisé ce montage pour l'étude de la série jaune de la cuprite, située aux environs de $5750 \AA$ à $4 \mathrm{~K}$, avec divers types d'émulsions photographiques.

La figure 2 représente un spectre et sa dérivée enre-

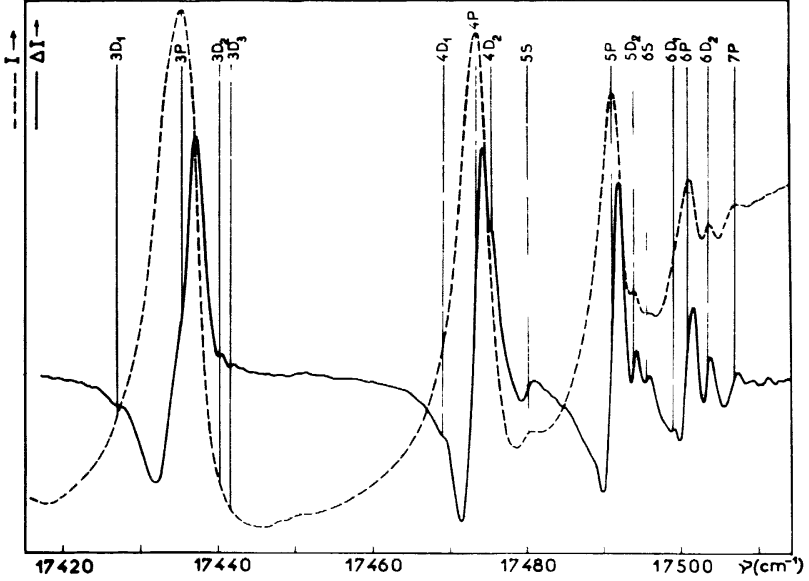

FIG. 2. - Densitométrages normal $(I)$ et différentiel $(\Delta I)$ donnant des représentations du spectre d'absorption du $\mathrm{Cu}_{2} \mathrm{O}$ et de sa dérivée par rapport à la longueur d'onde. Les unités sont arbitraires. L'épaisseur de l'échantillon est de $30 \mu \mathrm{m}$. Le spectre a été obtenu avec un temps de pose de $15 \mathrm{~min}$. et la durée totale du densitométrage est de $3 \mathrm{~min}$. Le même spectre a été étudié avec un montage spectrophotométrique, utilisant le même appareil dispersif que dans le présent travail, et permettant une modulation de la longueur d'onde [6]. Avec une résolution six fois moindre que dans le cas présent, le temps nécessaire à l'exploration du spectre est alors de 30 min. environ.

gistrés à partir de plaques Illford R $50\left({ }^{1}\right)$ avec des fentes de $50 \mu \mathrm{m}$. La figure 3 montre des résultats obtenus à partir de films Gevaert Scientia 45 C $62\left({ }^{1}\right)$.

Le spectre de la figure 2 est composé de raies $\mathrm{nP}$, formant une série hydrogénoïde et de raies moins intenses (raies de structure fine) à caractère $\mathrm{S}$ ou $\mathrm{D}$. Certaines de ces raies ne sont observables que sur la dérivée du spectre, comme les raies $\mathrm{D}_{1}$ par exemple, qui n'avaient encore jamais été observées. D'autres sont observables sur les deux enregistrements, mais leur position se détermine plus aisément sur le spectre dérivé.

La figure 3 donne un exemple de décomposition de la raie $4 \mathrm{P}$ en présence d'un champ magnétique dans la géométrie de Voigt. L'intensité des raies $\mathrm{D}$ diminuant considérablement en présence du champ, elles ne sont plus observables à $14,5 \mathrm{kG}$.

En plus de la raie notée $4 \mathrm{P}_{1}$, observée à champ nul, une raie, notée $4 \mathrm{P}_{3}$ apparaît à partir de $10 \mathrm{kG}$ et augmente en intensité, jusqu'à égaler celle de $4 \mathrm{P}_{1}$ à $21,5 \mathrm{kG}$. Seul, le spectre dérivé permet de bien apprécier ce comportement. Il nous a permis de constater que la position de $4 \mathrm{P}_{3}$, extrapolée à champ nul, diffère de celle de $4 P_{1}$. Ce résultat est fondamental pour déterminer l'origine de la raie $4 \mathrm{P}_{3}$ [5].

(1) Indications des fabricants :

— Illford R 50 : grain très fin et contraste très élevé.

- Gevaert Scientia 45 C $62\left\{\begin{array}{l}\text { résolution : } 180 \text { lignes } / \mathrm{mm} \text {, } \\ \text { contraste : } \simeq 2,5 .\end{array}\right.$ sensibilité : 12 à 15 DIN. 


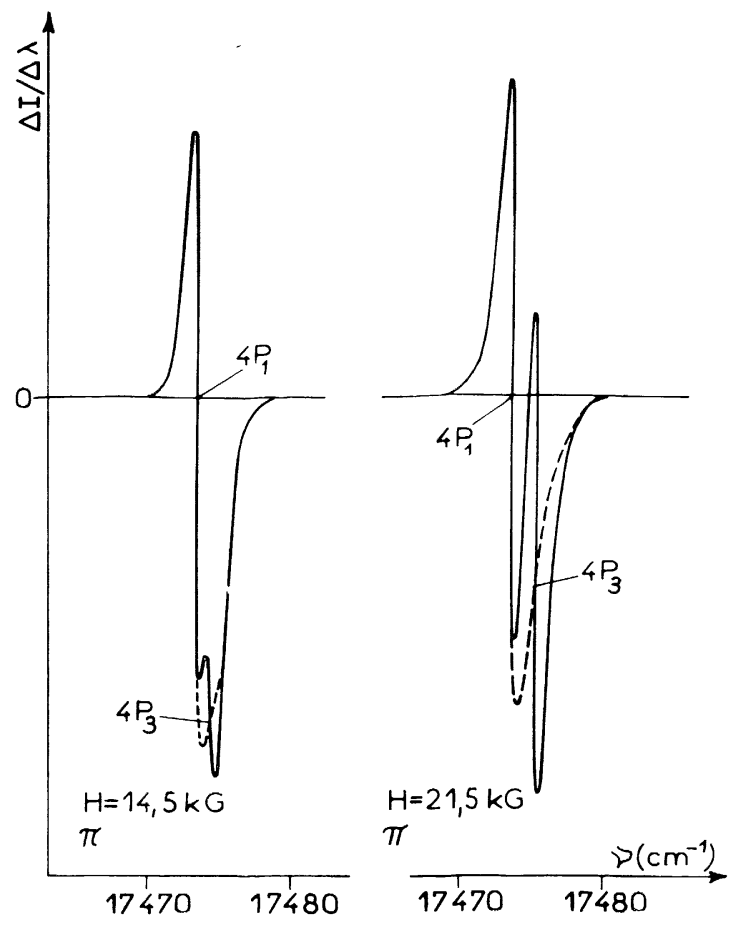

FIG. 3. - Densitométrage différentiel (en unités arbitraires) de la raie d'absorption $4 \mathrm{P}$ de la série jaune de la cuprite. L'échantillon est étudié en présence d'un champ magnétique perpendiculaire à la direction d'observation, et dans la polarisation $\pi$. L'épaisseur de l'échantillon est de $30 \mu \mathrm{m}$.

6. Utilisation du densitomètre en chromatographie. Comme nous l'avons noté au paragraphe 1, le densitomètre est d'usage courant pour l'analyse des chromatogrammes sur couches minces et des électrophorèses. De tels densitomètres ne diffèrent pas fondamentalement de ceux que l'on utilise en spectrographie, mais les échantillons sont toutefois généralement éclairés en lumière colorée, dans une bande spectrale adaptée à chaque cas particulier.

Nous avons étudié deux types d'échantillons. Dans le premier cas (électrophorèse), ceux-ci ont la forme de cylindres d'un centimètre de diamètre et d'une dizaine de centimètres de longueur. Ils sont composés d'un gel transparent, non biréfringent où l'on distingue un certain nombre de disques colorés (bandes) orientés perpendiculairement à l'axe du cylindre. La forme particulière de l'échantillon réduit la résolution effective de notre appareil à $200 \mu \mathrm{m}$, ce qui est toutefois largement suffisant dans le cas étudié. Les densitométrages différentiels obtenus sont de bonne qualité, le gel n'ayant pas de structure granulaire détectable. Ils permettent de localiser certaines bandes de faible intensité que l'on distingue très mal sur les densitométrages normaux. La bonne qualité optique des gels permet de penser qu'un densitomètre différentiel puisse résoudre un certain nombre de problèmes rencontrés en électrophorèse.

Nous avons également étudié des chromatogrammes sur couches minces. Le support est alors constitué d'une couche de silice en poudre, mélangée à un liant et étalée sur une lame de verre. Sur ce support laiteux, on observe, en transparence, des bandes parallèles et colorées.

La poudre de silice dépolarisant la lumière, nous avons été amenés à modifier notre appareil en prenant comme modulateur (Fig. 1) un polariseur tournant et en supprimant les analyseurs $A_{1}$ et $A_{2}$. Avec ce système, les fentes $F_{1}$ et $F_{2}$ sont éclairées alternativement. La détection se fait à l'aide de l'un des photodétecteurs qui délivre un signal différentiel alternatif dont l'intensité est proportionnelle à la quantité $I_{1}-I_{2}$ de la figure 1. Ce signal est mesuré par l'intermédiaire d'un amplificateur synchrone. Le photodétecteur délivre également un signal continu, proportionnel à $I_{1}+I_{2}$ et que l'on mesure à l'aide d'un électromètre. Ceci permet d'obtenir le spectre classique. Néanmoins, la résolution est moindre, pour ce spectre, que dans le cas du montage de la figure 1, car il est analysé, en fait, avec une fente dont la largeur est double de celle de $F_{1}$ ou $F_{2}\left({ }^{2}\right)$.

Sur les échantillons que nous avons étudiés, la silice présentait un grain important et les dérivées que nous avons obtenues n'ont, en conséquence, pas apporté de nouvelles informations. Il semble cependant que des mesures différentielles puissent être utiles, d'une part pour éliminer le rôle du fond continu d'absorption qui varie avec l'épaisseur non uniforme de la couche de silice et, d'autre part pour des mesures quantitatives de l'intensité intégrée des raies (ou bandes) absorbantes. Mais il serait alors nécessaire de faire une étude systématique de la forme analytique de ces raies, ce qui suppose des conditions expérimentales parfaitement contrôlables pour assurer une bonne reproductibilité des spectres.

7. Perspectives. - L'originalité du densitomètre décrit ci-dessus vient de l'utilisation d'une double fente, ce qui permet d'obtenir simultanément un spectre et sa dérivée avec une résolution optimum. L'optique que nous utilisons paraît adaptable sur certains densitomètres commerciaux. Par ailleurs, les performances de notre appareil pourraient être accrues, en fonction de besoins précis, par l'utilisation de certains procédés connus : adjonction d'un second faisceau lumineux permettant une compensation des instabilités de la source lumineuse, couplage des mouvements du porteplaque et de l'enregistreur, asservissement de la vitesse d'analyse à un programme extérieur ou à certaines caractéristiques des spectres étudiés, etc.

Enfin, en remplaçant les fentes utilisées pour les densitométrages par des trous circulaires et en utilisant une platine possédant deux degrés de liberté, on pourrait analyser une image à deux dimensions. Pour chaque point analysé, on obtiendrait à la fois l'absorption et sa dérivée, prise sur un axe d'orientation donnée.

(2) Le dispositif décrit ici pourra être utilisé pour tout support biréfringent. Nous n'avons rencontré qu'exceptionnellement de tels supports, dans le cas des films photographiques. 
La direction de cet axe, dans le plan de l'image, peut varier continûment si l'on fait tourner le prisme de Wollaston (Fig. 1). Cette propriété semble intéressante pour l'établissement de courbes d'iso-noircissements ou de courbes de plus grande pente.

8. Conclusion. - Le densitomètre décrit ici a été réalisé avec des moyens courants de laboratoire. Il offre, pour l'analyse normale d'un spectre, des performances comparables à celles des appareils usuels. La précision et la facilité des réglages en font un outil commode et fiable.

L'expérience nous a montré que les mesures différentielles qu'il permet d'effectuer apportent des renseignements très utiles lors d'une analyse spectrale fine. Nous avons pu, en particulier, détecter des raies d'absorption de très faible intensité et déterminer leur position avec précision. Les progrès réalisés actuellement dans le domaine des émulsions photographiques destinées aux hologrammes (plaques dites holographiques, à très grande résolution) permettent d'espérer une amélioration très sensible de ses performances.

Un densitomètre différentiel pourra être avantageusement utilisé dans tous les cas où l'image que l'on analyse n'a pas un caractère granulaire trop prononcé. Comparé à un appareil classique, il donne davantage d'informations, sans allonger ni les temps de réglages, ni les temps d'analyse spectrale. Son utilisation devrait donc se généraliser.

Remerciements. - Nous tenons à remercier le Professeur S. Nikitine qui nous a constamment encouragés et qui nous a suggéré certaines expériences. Nous remercions également MM. P. L. Wendel et C. Noguet pour leurs conseils techniques.

Les chromatogrammes sur plaque et les échantillons d'électrophorèse nous ont été prêtés par MM. G. Rebel, J. P. Fuchs et G. Hildwein du Centre de Neurochimie du C. N. R. S. (Directeur: M. P. Mandel) de Strasbourg. Nous les en remercions vivement.

\section{Bibliographie}

[1] Stauffer, F. R. et SaKai, H., Appl. Opt. 7 (1968) 61.

[2] Cardona, M., Modulation Spectroscopy (Acad. Press) 1969.

[3] Shaklee, K. L. et Rowe, J. E., Appl. Opt. 9 (1970) 627.

[4] Aramu, F. et Rucci, A., Rev. Sci. Instrum. 37 (1966) 1696.
[5] Merle, J. C., Wecker, C., Daunois, A., Deiss, J. L. et Nikitine, S., Surface Science 37 (1973) 347.

[6] Daunois, A., Merle, J. C., Deiss, J. L. and Nikitine, S., Phys. Stat. Sol. (b) 50 (1972) 691. 